G. Faltings

Nagoya Math. J.

Vol. 77 (1980), 99-106

\title{
A CONTRIBUTION TO THE THEORY OF FORMAL MEROMORPHIC FUNCTIONS
}

\author{
GERD FALTINGS
}

In my paper [F3] I more or less explicitly conjectured that if $A$ is a complete local integral domain with maximal ideal $\mathfrak{m}$ and if $I=\left(t_{1}, \cdots\right.$, $\left.t_{n}\right)$ is an ideal in $A$ with $n \leqslant \operatorname{dim}(A)-2$, then $\operatorname{Spec}(A / I)-\mathfrak{m}$ is $G 3$ in $\operatorname{Spec}(A)-\mathfrak{m}$. This will be proved in this paper.

Unfortunately the algebraisation-theorems for subsheaves in [F3] make great difficulties in this context, and I only can prove similar theorems, where I deal not with complete local rings but with a different category which will be explained in detail later. At least our theorems are sufficient to deal with projective varieties.

As corollary I obtain for example a strong generalisation of the G3theorems in [S].

This work was supported by the Deutsche Forschungsgemeinschaft.

\section{$\S 1 . \quad$ Notations}

All rings are commutative, noetherian and have a unit. If $A$ is such a ring, $\mathscr{I} \subseteq \mathfrak{a} \subseteq A$ two ideals, such that $A$ is complete in the $\mathscr{I}$-adic topology, then $X:=\operatorname{Spec}(A), Y:=\operatorname{Spec}(A / \mathscr{I}) \subseteq X, Z:=\operatorname{Spec}(A / \mathfrak{a}) \subseteq Y$, $U:=X-Z, \hat{X}:=\operatorname{Spf}(A)=$ formal completion of $X$ along $Y$ and $\hat{U}:=$ formal completion of $U$ along $U \cap Y$. If anywhere in this paper there appears a ring $A$ with ideals $\mathscr{I}$ and $a$, these notations are used without further comment. If there occur other rings $A_{1}, A_{2}, \cdots$ with ideals $\mathscr{I}_{1}, \mathscr{I}_{2}, \cdots$ and $\mathfrak{a}_{1}, \mathfrak{a}_{2}, \cdots$, then $X_{1}, X_{2}, \cdots, U_{1}, U_{2}, \cdots$ etc. are defined according to these data in the same way as above.

If $f$ moves in a system of generators for $\mathfrak{a} / \mathscr{I}$, then $\hat{U}$ can be glued together from the $\operatorname{Spf}\left(A_{(f)}\right)$, where $A_{(f)}$ is the $\mathscr{I}$-adic completion of the ordinary localisation $A_{(f)}$. If $M$ is a finitely generated $A$-module, then $M$ induces a coherent sheaf on $\hat{X}$ and $\hat{U}$. Sheaves arising in this way are

Received December 12, 1978. 
called algebraic. So over $\hat{X}$ any coherent sheaf is algebraic.

The elementary commutative algebra of $[\mathrm{M}]$ is used without explicit comment. Let $A, \mathscr{I}, \mathfrak{a}$ be as above, with $A$ local integral and universal catenary. If $X^{\prime}$ is the projective $A$-scheme obtained by blowing up $Y$ in $X$, then some easy calculations as in [F2], Lemma 2 show that $X^{\prime}$ is integral and that for all closed points $x \in X^{\prime}$ we have $\operatorname{dim}\left(\mathcal{O}_{x, X^{\prime}}\right)=\operatorname{dim}(A)$.

In an abuse of language a ring $A$ is called unmixed if for all maximal ideals $\mathfrak{m}$ of $A$ and all minimal prime ideals $\mathfrak{p} \subseteq \mathfrak{m}$ we have $\operatorname{dim}\left((A / \mathfrak{p})_{\mathfrak{m}}\right)$ $=\operatorname{dim}(A)$. By the above we see without any difficulty that for a universal catenary unmixed ring $A$ any ring of an affine piece of the scheme obtained by blowing up an ideal in $A$ has the same properties and the same dimension as $A$.

\section{§2. The G3-Theorem}

Theorem 1. Let $A$ be an unmixed integral domain which has a dualising complex. Let $\mathscr{I} \subseteq \mathfrak{a} \subseteq A$ be two ideals such that $I=\left(t_{1}, \cdots, t_{n}\right)$ with $n \leqslant \operatorname{dim}(A)-\operatorname{dim}(A / \mathfrak{a})-2$.

Then every formal meromorphic function on $\hat{U}$ is contained in $Q(A)$, the field of fractions of $A$. (equivalently, $U \cap Y$ is $G 3$ in $U$. )

Proof. Let $X^{\prime}$ be the scheme obtained by blowing up $Y \subseteq X$. Then $X^{\prime}$ is integral. If $B$ is the affine ring of one of the affine pieces of $X^{\prime}$, consider the ring $\cup(B: \mathfrak{b})=\cap B_{\mathfrak{p}} \subseteq Q(A)$, where $\mathfrak{b}$ runs through all ideals of $B$ with height $(\mathfrak{b}) \geqslant 2$ and $\mathfrak{p}$ through all prime ideals of $B$ with height $(\mathfrak{p}) \leq 1$. By [F1], Satz 2 this ring is finite over $B$, and for the various $B$ 's these rings glue together to a finite, quasicoherent $\mathcal{O}_{X^{\prime}}$-algebra. Let $W$ be the scheme associated with this algebra and $p$ the natural mapping $p: W \rightarrow X$. Then $W$ is integral, $p$ is proper and dominant, for all closed points $x \in W \operatorname{dim}\left(\mathcal{O}_{X, W}\right)=\operatorname{dim}(X)$ and all local rings of $W$ satisfy the $S_{2}$ condition. As $X^{\prime}$ can be imbedded in $X \times \boldsymbol{P}^{n-1}$ and as $p^{-1}(Z)$ is finite over the preimage of $Z$ in $X^{\prime}$, we get that $\operatorname{dim}\left(p^{-1}(Z)\right) \leqslant \operatorname{dim}(Z)+n-1$ $\leqslant \operatorname{dim}(X)-3$.

Let $f$ be a formal meromorphic function on $\hat{U}$. Let $V=p^{-1}(U) \subseteq W$ and $\hat{V}$ and $\hat{W}$ the formal schemes obtained by completing along $p^{-1}(Y)$. Then $f$ induces a formal meromorphic function on $\hat{V}$, and $I$ show that $f$ can be extended to a formal meromorphic function on $\hat{W}$. For this consider an affine piece $\operatorname{Spf}\left(A_{1}\right)$ of $\hat{W}$, where $A_{1}$ is the completion of an affine 
ring of $W$ in the $\mathscr{I}$-adic topology. Then all maximal ideals of $A_{1}$ have height $\geqslant \operatorname{dim}(X)$ and $Z_{1}=\operatorname{Spf}\left(A_{1}\right) \cap p^{-1}(Z)$ is defined by an ideal $\mathfrak{a}_{1} \subseteq A_{1}$ with $\operatorname{dim}\left(A_{1} / \mathfrak{a}_{1}\right) \leqslant \operatorname{dim}(X)-3$. It is also wellknown that $A_{1}$ is unmixed, and we may assume that $\mathscr{I} \cdot A_{1}=\mathscr{I}_{1}$ is generated by a single element $t \in A$ with $t \in a_{1}$. We have to prove, that $f \in Q\left(A_{1}\right)$ (=total ring of fractions of $A_{1}$ ).

Let $\mathscr{J} \subseteq \mathcal{O}_{\hat{U}_{1}}$ be the ideal defined by $\mathscr{J}=\mathcal{O}_{\hat{\theta}_{1}} \cap f^{-1} \cdot \mathcal{O}_{\hat{\theta}_{1}}=\left\{x \mid x \in \mathcal{O}_{\hat{U}_{1}}\right.$ and $\left.f \cdot x \in \mathcal{O}_{\hat{\theta}_{1}}\right\}$. After multiplying $f$ with some power of $t$, we may assume that $t$ is not a divisor of zero in $\mathcal{O}_{\hat{U}_{1}} / \mathscr{J}$. (Here we use that $A_{1}$ and the local rings in $\mathcal{O}_{\hat{U}_{1}}$ fulfill $S_{2}$.)

$f$ and 1 both define global sections of the coherent $\mathcal{O}_{\hat{v}_{1}}$-sheaf $\mathscr{F}$ $=\mathscr{H}_{o m_{0} \hat{U}_{1}}\left(\mathscr{J}, \mathcal{O}_{\hat{U}_{1}}\right)$. By the hypotheses on $\mathcal{O}_{\hat{v}_{1}} / \mathscr{J}$ we get an inclusion

$$
\mathscr{J} \mid t \cdot \mathscr{J} \subseteq \mathcal{O}_{\hat{U}_{1}} / t \cdot \mathcal{O}_{\hat{U}_{1}},
$$

and we have an exact sequence

$$
0 \rightarrow \Gamma\left(\hat{U}_{1}, \mathscr{F}\right) \rightarrow \Gamma\left(\hat{U}_{1}, \mathscr{F}\right) \rightarrow \Gamma(\hat{U}, \mathscr{F} \mid t \cdot \mathscr{F}),
$$

where the last module is contained in $\Gamma\left(\hat{U}_{1}, \mathscr{H}_{\text {omo }_{\hat{v}_{1}}}\left(\mathscr{J} / t \cdot \mathscr{J}, \mathscr{O}_{\hat{U}_{1}} / t \cdot \mathcal{O}_{\hat{U}_{1}}\right)\right.$.

The last module is finitely generated by [F1], Satz 2, and by the hypotheses on $\mathscr{J}$ it is easy, to see, that for all minimal primeoverideals $\mathfrak{p}$ of $t \cdot A_{1}$ its localisation in $\mathfrak{p}$ is a free $A_{1}$-module generated by the image of the natural inclusion $\mathscr{J} \subseteq \mathcal{O}_{\hat{\theta}_{1}}$, which is an element of $\Gamma\left(\hat{U}_{1}, \mathscr{F}\right)$.

Let $M:=\Gamma\left(\hat{U}_{1}, \mathscr{F}\right) . \quad$ As $M$ is separated in the $t$-adic topology and as $M / t \cdot M$ is a finitely generated $A_{1}$-module, $M$ is finitely generated over $A_{1}$. By assigning to every $a \in A_{1}$ the mapping $\mathscr{J} \rightarrow \mathcal{O}_{\hat{U}_{1}}$ defined by the multiplication with $a$, we get an injection $A_{1} \subseteq M$. As for all minimal primeoverideals $\mathfrak{p}$ of $t \cdot A_{1}$ the localisation of this mapping in $\mathfrak{p}$ induces a surjection $A_{1, \mathfrak{p}} \rightarrow\left(\Gamma\left(\hat{U}_{1}, \mathscr{H}_{\mathrm{om}_{\mathrm{O}_{0}}}\left(\mathscr{J} / t \cdot \mathscr{J}, \mathcal{O}_{\hat{\theta}_{1}} / t \cdot \mathcal{O}_{\hat{U}_{1}}\right)\right)_{\mathfrak{p}} \supseteq(M / t \cdot M)_{\mathfrak{p}}\right.$, we get that the localisation of this mapping in $\mathfrak{p}$ is an isomorphism. Therefore the localisations of these mappings in $\mathfrak{q}$ for all minimal primeideals $\mathfrak{q}$ in $A_{\mathrm{r}}$ are also isomorphic. As $f$ defines an element of $M$, we then obtain that $f \in Q\left(A_{1}\right)$. From now on we view $f$ as a global meromorphic function on $\hat{W}$. By applying the algebraisation-theorem [G], Th. 5.1.4 to the graph of $f$, we see that in fact $f$ as a meromorphic function on $W$. As the projection $p$ is birational $f$ is in fact an element of $Q(A)$.

Copying the proof of [F3], Theorem 2, we obtain the following corollaries: 
CoRollary 2. Let $k$ be a field, $\mathfrak{p}, \mathfrak{q} \subseteq k\left[\left[T_{1}, \cdots, T_{n}\right]\right]$ primeideals, such that $k$ is algebraically closed in $Q\left(k\left[\left[T_{1}, \cdots, T_{n}\right]\right] / \mathfrak{p}\right)$ and such that $Q\left(k\left[\left[T_{1}\right.\right.\right.$, $\left.\left.\left.\cdots, T_{n}\right]\right] / \mathfrak{p}\right)$ is separable over $k$. If $\mathfrak{a} \subseteq A:=k\left[\left[T_{1}, \cdots, T_{n}\right]\right] / \mathfrak{q}$ is an ideal containing $\mathscr{I}:=\mathfrak{p} \cdot A$ with

$$
\operatorname{dim}(A / \mathfrak{a})+n+2 \leqslant \operatorname{dim}\left(k\left[\left[T_{1}, \cdots, T_{n}\right]\right] / \mathfrak{p}\right)+\operatorname{dim}(A), \hat{U} \text { is } G 3 .
$$

Corollary 3. Let $k$ be a field, $X, Y \subseteq \boldsymbol{P}_{k}^{n}$ irreducible subschemes with $X$ geometrically integral. Let $Z \subseteq X \cap Y$ be a closed subscheme.

If $\operatorname{dim}(X)+\operatorname{dim}(Y) \geqslant n+\operatorname{dim}(Z)+2($ where for $Z=\varnothing \operatorname{dim}(Z):=-1)$, then $(X \cap Y)-Z$ is $G 3$ in $Y-Z$.

This includes the results about G3 in [S].

\section{§3. Algebraisation of subsheaves}

THEOREM 4. Let $A$ be an unmixed ring with two ideals $\mathscr{I} \subseteq \mathfrak{a} \subseteq A$, such that $\mathscr{I}$ is generated by $n$ elements and such that $A$ is complete in the $\mathscr{I}$-adic topology. Suppose $A$ contains a field $k$, such that $A / \mathscr{I}$ is a finitely generated k-algebra.

Let $M$ be a finitely generated A-module with associated coherent formal sheaf $\mathscr{F}$ on $\hat{U}$. Let $\mathscr{G} \subseteq \mathscr{F}$ be a coherent formal subsheaf, such that for all $f \in \mathfrak{a}$ and all $\mathfrak{p} \in \operatorname{Ass}_{A_{(f)}}\left((\mathscr{F} / \mathscr{G}) \otimes_{o \mathfrak{O}} A_{(f)}\right) \operatorname{dim}\left(A_{(f)} / \mathfrak{p}\right) \geqslant \operatorname{dim}(A / \mathfrak{a})+n+2$. Then there exists a submodule $N$ of $M$, such that $\mathscr{G}$ is the coherent sheaf associated with $N$.

Proof. I first remark that for an unmixed ring $A$ which has a dualising complex any integral quotient and any completion in an $\mathscr{I}$-adic topology fulfills the same conditions. It is wellknown, that for our ring $A$ these conditions are fulfilled, and that the same in true for all the $A_{(f)}$.

$I$ first prove the theorem for $n=1$, so that $\mathscr{I}$ is generated by an element $t \in \mathfrak{a}$. Using the "primary decomposition" of [F3], Lemma 1, we may assume that for all the $\mathfrak{p}$ listed in the inequality in our assumption $\operatorname{dim}\left(A_{(f)} / \mathfrak{p}\right)=s=$ constant independent of $\mathfrak{p}$. We may as well separate the primary components belonging to $\mathfrak{p}$ 's which contain $t$ from the rest and therefore assume that either $t$ is not a divisor of zero in $\mathscr{F} / \mathscr{G}$ or that $\mathscr{F} \mid \mathscr{G}$ is annihilated by some power of $t$. In the second case our theorem is trivial, so that from now on we assume that $t$ is regular in $\mathscr{F} / \mathscr{G}$.

Then $\mathscr{F} /(\mathscr{G}+t \cdot \mathscr{F})$ is algebraic and can be algebraisized by a module of dimension $s-1$. By Noether's normalisation theorem we can choose 
$x_{1}, \cdots, x_{s-1} \in A$ such that $A / \operatorname{Ann}(\mathscr{F} /(\mathscr{G}+t \cdot \mathscr{F}))$ is finite over $k\left[x_{1}, \cdots, x_{s-1}\right]$. Put $A_{1}:=k\left[x_{1}, \cdots, x_{s-1}\right][[t]] \subseteq A, \mathscr{I}_{1}:=t \cdot A_{1}$ and $\mathfrak{a}_{1}:=\mathfrak{a} \cap A_{1}$ and copy the proof of the analogous statement in [F3], Theorem 3.

Now let $\mathscr{I}$ be arbitrary again. Let $X^{\prime}$ be the projective $A$-scheme obtained by blowing up $Y \subseteq X, p$ the canonical mapping $X^{\prime} \rightarrow X$ and $Y^{\prime}:=p^{-1}(Y), Z^{\prime}:=p^{-1}(Z)$. Then $\operatorname{dim}\left(Z^{\prime}\right) \leqslant \operatorname{dim}(Z)+n-1$. Let $U^{\prime}:=X^{\prime}$ $-Z^{\prime}, \hat{X}^{\prime}$ and $\hat{U}^{\prime}$ the formal completions along $Y^{\prime}$. We may again assume that $\operatorname{dim}\left(A_{(f)} / \mathfrak{p}\right)=s=$ constant for all the $\mathfrak{p}$ 's in our assumption.

Let $\mathscr{J} \subseteq \mathcal{O}_{U}$, be the intersection of all primary components of dimension $\geqslant s$ of $\left(\operatorname{Ann}_{\mathscr{O} \hat{U}}(\mathscr{F} / \mathscr{G})\right) \cdot \mathcal{O}_{\hat{0}} \cdot$

By some local calculation it is seen that $\operatorname{Supp}\left(\mathcal{O}_{\hat{\theta}} \mid \mathscr{J}\right) \subseteq \hat{U}^{\prime}$ is mapped by $p$ onto $\operatorname{Supp}(\mathscr{F} / \mathscr{G}) \subseteq \hat{U}$. By our theorem for the case $n=1 \mathscr{J}$ can be continued to a coherent ideal on $\hat{X}^{\prime}$, which by [G], Th. 5.1.4 is given by an ideal in $\hat{X}$. Finally we get an ideal $\mathfrak{b} \subseteq A$ with $\operatorname{dim}(A / \mathfrak{b})=s$, such that $\mathfrak{b}$ annihilates $\mathscr{F} / \mathscr{G}$.

We then may reduce to the case where $\mathscr{F} / \mathscr{G}$ is annihilated by a primeideal $\mathfrak{p} \subseteq A$ with $\operatorname{dim}(A / \mathfrak{p})=s$, and the proof may be finished similar to [F3], Theorem 3 using the fact that $A / \mathfrak{p}$ is $G 3$.

If we copy the proof of [F3] Theorem 3, where the complete tensor product $A \hat{\otimes}_{k}(A / \mathscr{I})$ must be replaced by the ordinary tensor product $A \otimes_{k}(A / \mathscr{I})$ (which makes a lot of things easier than there), we get the following corollaries, where in the second one we consider the completion of the ring of the affine cone over a projective scheme in an $\mathscr{I}$-adic topology:

Corollary 5. Let $k$ be a field, $A$ the completion of $k\left[T_{1}, \cdots, T_{n}\right]$ in an $\mathscr{I}$-adic topology, where $\mathscr{I} \subseteq A$ is a primeideal, such that $k$ is algebraically closed in $Q(A / \mathscr{I})$ and such that $Q(A / \mathscr{I})$ is separable over $k$. Let $\mathfrak{a} \supseteq \mathscr{I}$ be another ideal. Suppose $M$ a finitely generated A-module, $\mathscr{F}$ the associated coherent formal sheaf on $\hat{U}$ and $\mathscr{G}$ a coherent subsheaf of $\mathscr{F}$, such that for all $f \in \mathfrak{a}$ and all

$\mathfrak{p} \in \operatorname{Ass}_{A_{(f)}}\left((\mathscr{F} / \mathscr{G}) \otimes_{\hat{O} \hat{U}} A_{(f)}\right) \quad \operatorname{dim}\left(A_{(f)} / \mathfrak{p}\right) \geqslant n+\operatorname{dim}(A / \mathfrak{a})-\operatorname{dim}(A / \mathscr{I})+2$.

Then there exists a submodule $N$ of $M$, such that $\mathscr{G}$ is the coherent sheaf associated with $N$.

CoRollary 6. Let $k$ be a field, $X \subseteq \boldsymbol{P}_{k}^{n}$ a geometrically integral subscheme and $Z \subseteq X$ a closed subscheme. Let $U:=\boldsymbol{P}_{k}^{n}-Z, \hat{U}:=$ formal completion 
of $U$ along $X-Z$. Suppose $\hat{Y} \subseteq \hat{U}$ is a closed formal subscheme, such that for all closed points $y \in \hat{Y}$ and all associated primeideals $\mathfrak{p}$ of $\mathcal{O}_{y, \hat{Y}}$ $\operatorname{dim}\left(\mathcal{O}_{y, \hat{Y}} / \mathfrak{p}\right) \geqslant n+\operatorname{dim}(Z)-\operatorname{dim}(X)+2 . \quad($ where again $\operatorname{dim}(\varnothing):=-1)$ Then there exists a closed subscheme $Y \subseteq U$, such that $\hat{Y}$ is the formal completion of $Y$.

\section{§4. G3 and cohomological dimension}

As the relation between $G 3$ and cohomological dimension does not seem to be quite obvious, I state the following result, where the notation $c d(X)$ for a noetherian scheme $X$ denotes the maximal $p$ with $H^{p}(X$, some coherent sheaf) $\neq 0$ :

CoRollaRy 7. Let $k$ be a field, $R=k\left[\left[T_{1}, \cdots, T_{n}\right]\right], \mathfrak{p}, \mathfrak{q} \subseteq R$ primeideals, such that $k$ is algebraically closed in $Q(R / \mathfrak{p})$ and such that $Q(R / \mathfrak{p})$ is separable over $k$. If $\operatorname{dim}(R / \mathfrak{p})+\operatorname{dim}(R / \mathfrak{q}) \geqslant n+2$, then

$$
\operatorname{cd}(\operatorname{Spec}(R / \mathfrak{q})-\operatorname{Spec}(R /(\mathfrak{p}+\mathfrak{q})))<\operatorname{dim}(R / \mathfrak{q})-2 .
$$

Proof. By Corollary 2 the formal completion of $\operatorname{Spec}(R / \mathfrak{q})-\{\mathfrak{m}\}$ $(\mathfrak{m}=$ maximal ideal of $R$ ) along $\operatorname{Spec}(R /(\mathfrak{p}+\mathfrak{q}))$ is $G 3$ and therefore the ring of global sections of this formal scheme is contained in $Q(R / \mathfrak{q})$. If $\mathfrak{x} \supset \mathfrak{q}$ is a primeideal of hight 1 in $R / \mathfrak{q}$, then by the dimension-inequalities of Serre $\operatorname{dim}(R /(\mathfrak{p}+\mathfrak{x}))>0$ and therefore $\mathfrak{p}+\mathfrak{x}$ is not $\mathfrak{m}$-primary. Hence every section of our formal scheme is contained in $(R / \mathfrak{q})_{\mathfrak{r}} \subseteq Q(R / \mathfrak{q})$. By [F1], Satz 2 the intersection of these $(R / \mathfrak{q})_{\mathrm{r}}$ is finite over $R / \mathfrak{q}$, and so is our ring of global sections. As the cokernel of the canonical morphism of $R / \mathfrak{q}$ in the ring of global sections of our formal scheme is equal to $\underset{\lim }{\longleftarrow}\left(H_{\mathrm{m}}^{1}\left(R /\left(\mathfrak{q}+\mathfrak{p}^{s}\right)\right)\right)$, we arrive at the conclusion that there exists an element $x \in R-\mathfrak{q}$ which annihilates this module. By local duality this module is paired with $\lim _{\longrightarrow}\left(\operatorname{Ext}_{R}^{n-1}\left(R /\left(\mathfrak{q}+p^{s}\right), R\right)\right.$, so that $x$ also annihilates the latter module.

Let $d=\operatorname{dim}(R / q)$. By Lichtenbaum's theorem $H_{p}^{d}(R / q)=0$ and for all $x \in R-\mathfrak{q} H_{\mathfrak{p}}^{d-1}(R /(\mathfrak{q}+x \cdot R))=0$. Therefore $H_{\mathfrak{p}}^{d-1}(\quad)$ is right-exact on $R / \mathfrak{q}$-modules and vanishes on all torsion- $R / \mathfrak{q}$-modules.

We have a spectral sequence

$$
E_{2}^{p, q}=H_{\mathfrak{p}}^{p}\left(\operatorname{Ext}_{R}^{q}(R / \mathfrak{q}, R)\right) \Rightarrow \underline{\lim }\left(\operatorname{Ext}_{R}^{p+q}\left(R /\left(\mathfrak{q}+\mathfrak{p}^{s}\right), R\right)\right)
$$

Here $E_{2}^{p, q}=0$ for $q<n-d$, and for $q>n-d$ the annihilator of $E_{2}^{p, q}$ is 
strictly larger than $\mathfrak{q}$. As we have seen above the latter property is also fulfilled by the $E_{\infty}^{p, q}$ for $p+q=n-1$. Therefore we may conclude that also the annihilator of $E_{2}^{d-1, n-d}=H_{\mathfrak{p}}^{d-1}\left(\operatorname{Ext}_{R}^{n-d}(R / \mathfrak{q}, R)\right)$ is strictly larger than $\mathfrak{q}$. Let $M=\operatorname{Ext}_{R}^{n-d}(R / \mathfrak{q}, R)$. Then $M$ is a torsionfree $R / \mathfrak{q}$-module of rank 1. Take an $x \in R-\mathfrak{q}$ which annihilates $H_{\mathfrak{p}}^{d-1}(M)$. Then by the long exact sequence of cohomology for $0 \longrightarrow M \stackrel{\times \cdot}{\longrightarrow} M \longrightarrow M / x \cdot M \longrightarrow 0$ we get an injection $H_{\mathfrak{p}}^{d-1}(M) \subseteq H_{\mathfrak{p}}^{d-1}(M / x \cdot M)$.

But the second module is 0 by Lichtenbaum's theorem, and so is the first. As there exists an injection $M G R / \mathfrak{q}$, we may again use the long exact sequence for cohomology and conclude that $H_{\mathfrak{p}}^{d-1}(R / \mathfrak{q})=0$ and therefore $H_{\mathfrak{p}}^{d-1}$ (any $R / \mathfrak{q}$-module) $=0$. This proves our corollary.

\section{§5. Applications to complex analytic geometry}

Let $X \subseteq \boldsymbol{P}_{\boldsymbol{C}}^{n}$ a closed irreducible analytic subset. Then $X$ is algebraic. If $U \supseteq X$ is an open set, then any complex analytic object on $U$ (as a meromorphic function or an analytic set) induces an analogous object on the complex analytic pendant of the formal completion of $\boldsymbol{P}_{\boldsymbol{C}}^{n}$ along $X$. By the usual GAGA-theory these objects come from formal algebraic objects. If these formal algebraic objects are algebraic in the usual sense then we may conclude that the analytic objects we started with can be continued to analytic objects on the whole of $\boldsymbol{P}_{C}^{n}$. Therefore we get the following corollaries:

Corollary 8. Let $X, Y \subseteq \boldsymbol{P}_{c}^{n}$ be closed irreducible analytic subsets, such that $\operatorname{dim}(X)+\operatorname{dim}(Y)>n$. If $U \subseteq Y$ is a connected open neighbourhood of $X \cap Y$ in $Y,(X \cap Y$ is connected by GAGA and Corollary 3), and if $f$ is a meromorphic function on $U$, then $f$ can be extended as a meromorphic function on $Y$.

Corollary 9. Let $X \subseteq \boldsymbol{P}_{\boldsymbol{C}}^{n}$ be a closed irreducible analytic subset, $U \supseteq X$ an open neighbourhood of $X$ in $\boldsymbol{P}_{C}^{n}$. If $A \subseteq U$ is a closed analytic subset, all whose irreducible components have a dimension $>n-\operatorname{dim}(X)$, then there exists a closed analytic subset $B \subseteq \boldsymbol{P}_{C}^{n}$ and an open neighbourhood $V \subseteq U$ of $X$, such that $A \cap V=B \cap V$.

\section{REFERENCES}

[F1] G. Faltings, Ueber die Annulatoren lokaler Kohomologiegruppen, Archiv der Math. 30 (1978), 473-476. 
[F2] G. Faltings Ueber Macaulayfizierung, Math. Annalen 238 (1978), 175-192.

[F3] G. Faltings, Some Theorems about Formal Functions, to appear.

[G] A. Grothendieck, Éléments de Géometrie Algébrique, Fasc. III, 1, Pub. Math. 11 (1961).

[M] H. Matsumura, Commutative Algebra, Benjamin, New York 1970.

[S] R. Speiser, Cohomological Dimension of Non-Complete Hypersurfaces, Inventiones math. 21 (1973), 143-150.

Dept. of Math.

Harvard University

1 Oxford Street

Cambridge, $M A 02138$

USA

and

Math. Inst. der Univ. Muenster

Roxeler Str. 64

4400 Muenster

Germany 